\title{
Coping style at diagnosis and its association with subsequent health-related quality of life in women with breast cancer: a 3-year follow-up study
}

Soo Hyun Kim, RN, PhD

Department of Nursing, Inha University, Incheon, South Korea

\section{INTRODUCTION}

Approximately $30 \%$ of breast cancer survivors (BCSs) reported adjustment difficulties, with about $22 \%$ demonstrating serious deteriorations in health-related quality of life (HRQOL). To facilitate the adjustment process and enhance the HRQOL of BCSs, researchers have been striving to identify the associated psychosocial factors.

Coping style is one important factor associated with HRQOL among BCSs, that is crucial to the adjustment process. Previous researches showed that adaptive coping styles are associated with better HRQOL, while maladaptive coping styles are associated with worse HRQOL.

The relationship between coping styles and psychological outcome for BCSs has been well documented, however, little is known about associations between coping style at diagnosis and long-term HRQOL. Furthermore, most studies were conducted in western countries, with limited application to Asian countries.

Thus, this study was performed to identify coping style clusters of Korean women diagnosed with breast cancer and examine how coping style was associated with $\mathrm{HRQOL}$ for 3 years after diagnosis.

\section{PURPOSE OF THIS STUDY}

The purpose of this study was to generate distinct coping styles of women newly diagnosed with breast cancer and to examine the longitudinal associations between coping style at diagnosis and subsequent health-related quality of life (HRQOL) over 3 years.

\section{METHODS}

* Study design: A prospective cohort study. We measured study variables at diagnosis (baseline), and followed up at 1, 2, and 3 years after enrollment.

*articipants

- Inclusion criteria

- Women aged over 19 years

- Diagnosed with breast cancer within the past month

- Who had a plan for a cancer treatment

- Who could read and write Korean

- Exclusion criteria

- Had other cancer(s)

- Had a severe cognitive impairment

- Were undergoing cancer treatment

Measures

- Coping style: The Korean version of the Mini-Mental Adjustment to Cancer (Mini-MAC) (Kang et al., 2008). This has five subscales including Helpless-Hopeless $(\mathrm{HH})$, Anxious preoccupation (AP), Fighting spirit (FS), Cognitive Avoidance (CA), and Fatalism (FA)

- HRQOL: The Korean version of the European Organization for Research and Treatment of Cancer Quality of Life Questionnaire Core 30 (EORTC QLQ-C30) (Yun et al., 2004)

* Data analysis:

- Descriptive statistics, K-means cluster analysis, Analysis of covariate (ANCOVA)

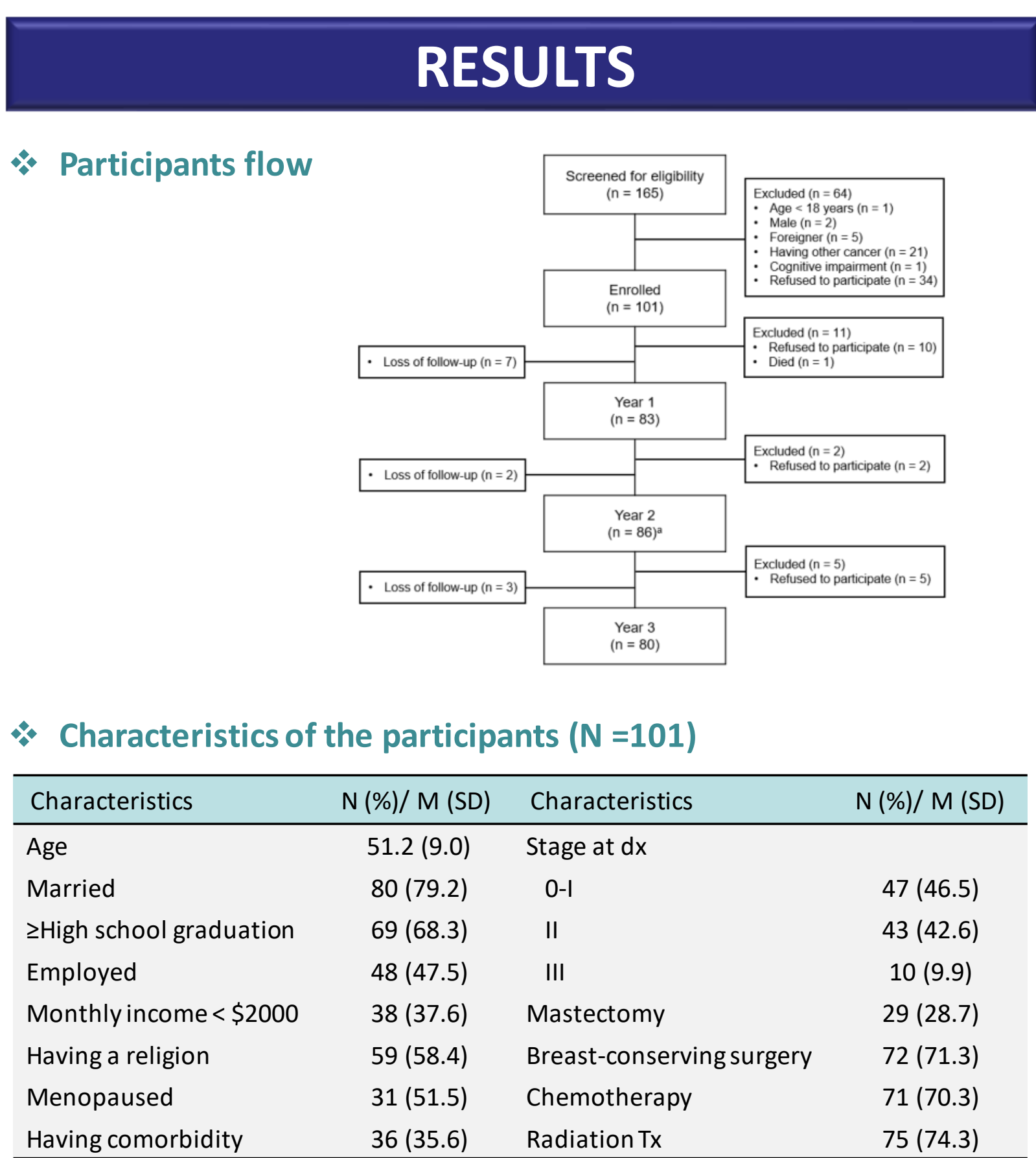

\section{- Coping styles identified in the study participants}

Based on the baseline data, we identified two coping style clusters. The first ("FS cluster") was characterized by higher scores on the FS subscale and lower scores on the HH and AP subscales ( $n=52,51.5 \%$ ); the second ("HHAP cluster") was characterized by higher scores on the $\mathrm{HH}$ and AP subscales and lower FS scores ( $n=49,48.5 \%)$.

- Differences in HRQOL by baseline coping style clusters across time

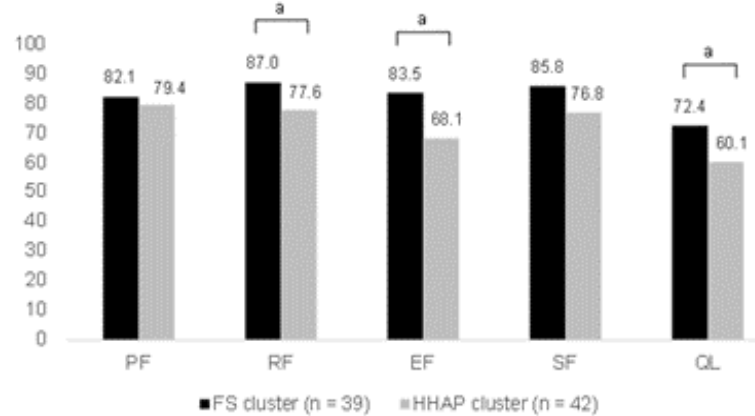

Year 1

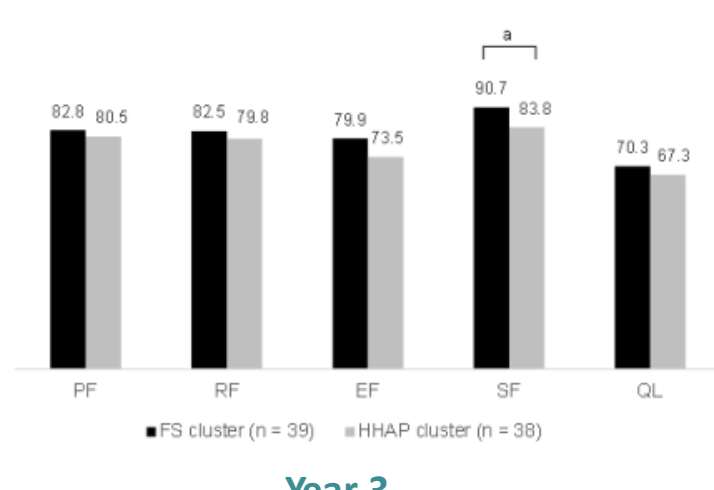

Year 3

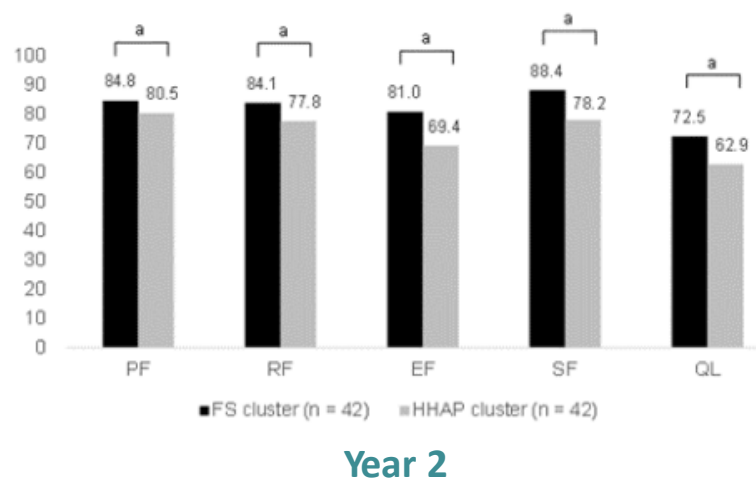

$E F=$ emotional function; $F S=$ fighting spirit HHAP, helpless/hopeless and anxious preoccupation; $\mathrm{PF}=$ physical function; $\mathrm{QL}=$ global quality of life; $\mathrm{RF}=$ role function; $\mathrm{SF}=$ social function

${ }^{\mathrm{a}} p<0.05$ adjusting for age, marital status, education level, income, religion, stage at diagnosis, recurrence, type of surgery, chemotherapy, radiation therapy, and antihormone therapy.

\section{CONCLUSIONS}

Maladaptive coping at diagnosis may predict poor $\mathrm{HRQOL}$ in patients with breast cancer. Regular screening of coping and interventions to improve poor coping style should be considered for at least 2 years from diagnosis. 